\title{
FUNDAMENTOS EPISTEMOLÓGICOS DE LA COMPETITIVIDAD TERRITORIAL PARA UN PROYECTO DE OBSERVATORIO URBANO-REGIONAL
}

\author{
EPISTEMOLOGICAL FUNDAMENTALS OF TERRITORIAL COMPETITIVENESS FOR AN \\ Urban and Regional Observatory Project
}

Tadeo Humberto Sanabria Artunduaga*

John Fredy Ramírez Ríos**

Recibido: 11 de enero de 2017

Aceptado: 25 de abril de 2017

\section{Resumen}

Se plantea una revisión de literatura alrededor del concepto de competitividad territorial con la finalidad de exponer sus fundamentos epistemológicos. A partir de allí, se propone una aproximación alternativa a la competitividad del territorio que lo conciba como unívoco y no objeto de fragmentación, construido a partir de fuerzas de cooperación competitiva que cumplen una función no solo económica, sino principalmente social. El análisis responde al proyecto de creación de una institución tipo Observatorio Urbano-Regional de la Competitividad Territorial en la Fundación Universidad de América, que a futuro permita la consolidación de teorías y evidencias empíricas sobre este concepto y su inclusión en políticas territoriales.

Palabras clave: competitividad territorial, epistemología, cooperación competitiva, observatorio urbano.

\section{Abstract}

A literature review is done around the territorial competitiviness with the purpose to disclose its epistemological fundamentals. From there, an alternative approach to competence on territory is suggested as unicity, shaped by cooperation forces which has a social function not just as an economic one. The analysis responds to the creation of an urban and regional observatory at the

\footnotetext{
*Arquitecto, magíster en Ordenamiento Urbano y Regional, magíster en Gestión y Valoración Urbana. Estudiante del Doctorado en Gestión y Valoración Urbana y Arquitectónica (Universidad Politécnica de Cataluña). Docente investigador, grupo de investigación Prospectiva Urbano-Territorial, Facultad de Educación Permanente y Avanzada, Fundación Universidad de América.Tadeo.sanabria@investigadores.uamerica.edu.co

** Sociólogo, magíster en Urbanismo. Docente investigador, grupo de investigación Prospectiva Urbano-Territorial, Facultad de Educación Permanente y Avanzada, Fundación Universidad de América.

john.ramirez@investigadores.uamerica.edu.co
} 
Universidad de América, which allows to generate empirical evidences, theories and public policies related to territorial competitiveness

Keywords: territorial competitiveness, epistemology, competitive cooperation, urban observatory.

\section{INTRODUCCIÓN}

En Colombia, independientemente de la afiliación política de los gobernadores y alcaldes y del tamaño y particularidades territoriales de los departamentos, municipios o centros urbanos, la competitividad territorial (CT) actúa como un discurso que sustenta tanto los programas, políticas y estrategias de los planes generales de desarrollo (los cuales se enfocan principalmente en el desarrollo social y económico), así como soporta los planes de ordenamiento territorial (que son los que se enfocan en la planeación espacial, también llamada planeación física). Algunas de esas estrategias parecieran formar parte del resorte de la planeación espacial; pero es difícil determinar hasta qué punto otras estrategias pertenecen más a la órbita de la planeación social o económica o la planeación regional, o se asocian con el también discutido ámbito de la planeación local o comunitaria.

Existen vacíos y confusiones alrededor de los diversos significados que puede tener la noción de CT en Colombia. Algunos trabajos recientes, como el de Ramírez (2015) o el de García y Acevedo (2010), intentan descifrar los alcances teóricos y empíricos de esta noción en el ámbito local, pero desde una perspectiva claramente macroeconómica. Hay análisis que desde una perspectiva crítica intentan mostrar que esta noción no hace alusión sino a otro de los muchos mecanismos de acumulación y expropiación capitalista; así, por ejemplo, Buitrago (2015) afirma que una plataforma territorial de competitividad debe ser entendida como "un sistema de territorialidades que articula áreas periféricas al sistema global capitalista, para entender los procesos de expoliación de la naturaleza y el abuso, por parte del Estado y los capitalistas, sobre las sociedades locales" (p. 238).

En ambas aproximaciones al concepto de CT se invisibilizan las dinámicas territoriales, que no solo tienen que ver con los flujos de los factores productivos como capital y trabajo, sino con la transferencia de otros capitales y recursos que no siempre responden a lógicas de extracción o explotación y que pueden estar dentro del empoderamiento y la cooperación. Es esa visión más integral de la CT la que da lugar a este artículo, en el que se desarrolla los fundamentos epistemológicos del concepto de competitividad territorial (CT) y que tiene como objetivo fundamentar teóricamente el desarrollo de un Observatorio Urbano-Regional de la Competitividad Territorial (OUCT) en la Fundación Universidad de América.

El proyecto se inscribe en la línea de investigación Ciudades Competitivas del grupo de investigación Prospectiva Urbano-Territorial. Desde el 2014, este grupo ha investigado sobre el concepto de competitividad territorial (Ramírez y Alzate, 2016a; Ramírez y Alzate, 2016b; Sanabria, Bolaños y Ramírez, 2015), con la finalidad de identificar las variables que permitan su medición y operacionalización en una institución tipo observatorio urbano (OU); así como también su inclusión en lineamientos de ordenamiento territorial. Variables que no solo incluyan las dimensiones económicas tradicionales del desarrollo del territorio, sino otras dimensiones que recojan los últimos aportes que la literatura internacional viene haciendo al respecto, por ejemplo: grados de transparencia territorial, institucionalidad territorial, facilidad para los negocios en los territorios, innovación, emprenderismo, gobernanza, etc.

Como el proyecto de OUCT de la Fundación Universidad de América busca inaugurar en Colombia un observatorio de lo competitivo del territorio, se hace indispensable empezar a definir qué tipo de concepto de la CT se medirá e intervendrá. Para responder a ese cuestionamiento se 
plantea la revisión de literatura como un método de investigación que permite una clara contextualización de las apuestas discursivas que determinan la validez epistemológica del fenómeno que eventualmente abordaría el OU propuesto.

\section{MÉTodo}

Durante los procesos de formulación de los proyectos de investigación, la revisión de literatura suele entenderse como un apartado marginal. Las normas de trabajo de grado exigen que los estudiantes y/o profesores lo incluyan, pero existen confusiones sobre la esencia y alcance de esta exigencia. Generalmente, la revisión se reduce a una compilación de fichas con textos y autores que tratan un tema común; pero es habitual que no exista una interpretación que le dé coherencia y sentido a esa compilación.

Este artículo corto revisa la literatura alrededor del concepto de CT desde una apuesta interpretativa que indaga en los discursos que afirman o niegan la validez de su estatuto epistemológico. Es preciso plantear esta discusión porque un OU solo puede estudiar fenómenos que puedan ser abordados desde una mirada científica objetiva. La noción del "observar" en un OU va más allá del solo "mirar", porque no solo implica el "ver", sino también el "conocer" y el "reconocer" la existencia objetiva de un fenómeno, en este caso, el de la competencia entre territorios.

De ahí que si "observar" implica "conocer", entonces se deriva una relación donde existe un sujeto $(\mathrm{S})$ que conoce y un objeto $(\mathrm{O})$ que es conocido. En esa medida, la sola noción de "observatorio" se podría ver bien significada por medio de la clásica relación gnoseológica sujeto-objeto (S-O). Las nociones "observar" y "observatorio" no se reducen al simple "mirar desprevenido", sino que ambas son concebidas como acto e institución humanas, respectivamente, y materializan el acto de conocer con todas sus implicaciones filosóficas. Se concluye, desde este punto de vista, que un $\mathrm{OU}$ establece una relación de conocimiento de un $\mathrm{S}$ (observatorio) que mira, conoce y reconoce un $\mathrm{O}$, que, en este caso, es lo competitivo de lo territorial o la CT.

La noción de OU gana otra propiedad significativa: no solo implica el conocer lo competitivo del territorio, sino también el controlarlo. Se observará como este juego de análisis epistémico se relaciona con la conceptualización más reciente de la noción de CT. Cuando se habla de OU de la CT se parte de un principio de cosificación u objetivación del territorio que no es universalmente aceptado. Existen discursos de apoyo o rechazo académico al concepto de CT. Los académicos europeos, por lo general, han aceptado la posibilidad de que la competitividad se objetive y materialice en el territorio, para así reducir los niveles de abstracción de los modelos económicos abstractos neoclásicos; por su parte, en el ámbito académico norteamericano ha dominado la tendencia contraria, es decir, desvirtuar ese intento de cosificación del territorio.

Esa situación dificulta la construcción de consensos alrededor de las variables y de los modelos econométricos o espaciales que permiten la medición y operacionalización de la competitividad en los territorios. También condiciona la viabilidad de constituir un OUCT; sin embargo, ofrece la oportunidad de asumir una postura epistemológica que permita su fundación no solamente material, sino intelectual. En otras palabras, lo que importa en la formulación de un proyecto de OU no es asegurar la viabilidad de una infraestructura física que permita su potencial funcionamiento, sino justificar la validez epistemológica del fenómeno-objeto alrededor del cual se harán futuras observaciones, mediciones y recomendaciones de política. Los resultados de esta justificación (basada en la revisión de la literatura) se presentan y discuten a continuación. 


\section{REsultados de LA REVISIÓN DE LITERATURA}

\section{El discurso dominante de la competitividad territorial en Europa}

En Europa, desde comienzos del siglo XIX, es considerable la documentación que considera el territorio como capital básico de la competitividad urbana y regional. Conferencias, artículos académicos y reportes de organismos multilaterales que han aparecido sobre todo en España, Italia, Grecia, Hungría y Bulgaria promocionan la importancia de que los países fomenten en las regiones y ciudades (como si se trataran de un stock) una serie de políticas que apunten a inventariar, modelizar e intervenir todas aquellas dimensiones operacionalizables de los territorios que ocupan. De hecho, una mirada bibliométrica elemental demuestra que la mayoría de la producción científica asociada con las nociones de CT se ha producido en Europa, principalmente en Italia (Tóth, 2014). Igualmente, hay un aporte importante de latinoamericana y de algunos organismos multilaterales encargados de financiar el desarrollo social y económico de países en desarrollo.

De hecho:

[...] El concepto de capital territorial fue inicialmente más un concepto de política pública que uno científico. Al tiempo que su conceptualización se ha desarrollado, también esas condiciones iniciales han estado cambiando. De hecho, el estado actual de conocimiento y comprensión del capital territorial provee unas mejores bases para que académicos lancen investigación empírica sobre este, que lo pueden hacer los profesionales y políticos para establecer políticas a partir del mismo. ${ }^{1}$ (Tóth, 2014, pp. 8-10)

Roberto Camagni presentó en 2002 la ponencia "On the concept of territorial competitiveness: sound or misleading?”, con la que buscó justificar la validez epistemológica del concepto CT. Modelos complejos y avanzados de econometría especial se bosquejan allí para su cálculo. Dicha conceptualización se ha establecido para validar todo tipo de políticas, que van desde las tradicionales, interesadas en el desarrollo económico local, rural y regional, hasta las más recientes, que tienen ver con la consolidación de industrias culturales, creativas, el fomento del turismo y el mercadeo territorial. Esto se ha visto como el detonante principal de las prácticas que se derivan de una economía globalizada basada en el conocimiento y la innovación.

La literatura producida principalmente en Europa sobre la CT se ha centrado en revisar, por un lado, si es posible hablar de capital territorial, es decir, de homologar el territorio al concepto de capital; al respecto, Toth (2014) señala que aunque "el capital de lo territorial no se adecúa a muchas de las propiedades del capital, varios de sus componentes (como el capital cultural, humano o social) pueden ser convertidos"2 (cf. Bourdieu, 1986 about 'conversions')" (p. 2). Por otro lado, el mismo Tóth plantea que la CT se adecuó a un "contexto de política regional" (p. 2) justo después de que la Organisation for Economic Cooperation and Development (OECD) publicara en 2001 un reporte titulado OECD Territorial Outlook, y lo definiera como "el stock de bienes los cuales forman la base para el desarrollo endógeno de cada ciudad y región, así como también de las instituciones, maneras de toma de decisión y habilidades profesionales para hacer un mejor uso de esos bienes"3 (OECD, 2001, p. 13).

\footnotetext{
${ }_{1}^{1}$ The concept of territorial capital was initiated as a policy concept (rather than a scientific one). As the conceptualization was being developed, the conditions seem to have been changing; the current knowledge and understanding on territorial capital provide a better basis for scholars to launch empirical research than for practitioners to establish certain policy concepts [traducción de los autores].

${ }^{2}$ Territorial capital is not adequate for many properties of 'capital', several of its components (e. g., cultural capital, human capital, and social capital) can be converted [traducción de los autores].

3 The stock of assets which form the basis for endogenous development in each city and region, as well as to the institutions, modes of decision-making and professional skills to make best use of those assets [traducción de los autores].
} 
Allí el tema ha sido rigurosamente tratado desde la perspectiva del desarrollo de estrategias espaciales orientadas a la oferta (Camagni, Caragliu y Perucca, 2011) y se ha centrado en demostrar que el territorio puede ser tratado como una especie de mercancía: se puede inventariar, modelizar e intervenir en todas aquellas dimensiones cuantificables con el fin de hacerlo más competitivo. Tal corriente de pensamiento y de política económica establece dos supuestos fundamentales: 1) el territorio puede ser concebido como un capital, un asset con propiedades similares al de otros capitales físicos, financieros, sociales, intelectuales, etc., y 2) el territorio, como todo capital, puede ser objeto de planificación y gestión que lo hagan más productivo y eficiente (Tóth, 2014, p. 10).

Tóth (2014) resume algunas definiciones paradigmáticas de capital territorial:

"Un conjunto de bienes localizados -naturales, artificiales, humanos, organizacionales, relacionales y cognitivos- que aglutinan el potencial de cierto territorio" (Camagni, 2009) [...] "La cantidad entrelazada de diferentes formas de capital (o de los diferentes recursos) implicados, movilizados y activamente usados en (y reproducidos por) la economía regional y la sociedad"5 (van der Ploeg et al., 2008, p. 13) [...] "Un stock de recursos específicos de un lugar y disponibles para aquellos quienes viven y trabajan en el territorio. Esos recursos (materiales e inmateriales) son bienes comunes a una comunidad local" (Ventura et al., 2008, p. 160) [...] "El conjunto de los bienes locales los cuales, si son adecuadamente movilizados, proveen ventajas comparativas a quienes viven y trabajan alli" ${ }^{7}$ (Giaime, 2011, p. 9). (p. 2-3)

E1 planteamiento de que el territorio puede ser tratado como un capital tiene una razón económica de fondo que es preciso aclarar. Mientras los estados nacionales son capaces hacer más competitivas sus exportaciones por medio de mecanismos artificiales, como la devaluación intencional de la moneda o la reducción legal de cargas salariales y prestacionales, las regiones y ciudades no pueden hacer uso de tales estrategias macroeconómicas. Ellas solo pueden competir internacionalmente haciendo más atractivos sus territorios, facilitando material y espacialmente el desarrollo de actividades económicas en los espacios geográficos que están bajo su jurisdicción legal. En ese sentido, no hay otra manera de elevar sus índices de competitividad sino es por medio del mejoramiento continuo de sus recursos naturales, culturales, políticos y sociales, lo cual obliga a su necesaria cuantificación, medición y monitoreo .

Como se puede observar, universidades y académicos europeos son los abanderados del discurso actual de la CT, en un claro contexto de reactivación económica de regiones que se han quedado rezagadas o que están siendo amenazadas por otras zonas del mundo con políticas agresivas de desarrollo económico. Sin embargo, la academia americana está menos convencida de que exista efectivamente un concepto de CT o, por lo menos, un concepto de competitividad desligado enteramente de los factores clásicos macroeconómicos, factores que por décadas han servido para explicar las dinámicas modernas del comercio internacional.

\section{EL DISCURSO HETERODOXO SOBRE LA COMPETITIVIDAD TERRITORIAL EN ESTADOS UNIDOS}

La literatura en Estado Unidos y el Reino Unido sobre el tema es escasa. Por lo general, su producción teórica y crítica niega la relevancia científica de la CT. En este sentido, Brenner y Wachsmuth (2012) afirman: "las políticas de competitividad territorial tienen un carácter inherentemente

\footnotetext{
${ }^{4}$ A set of localized assets - natural, artificial, human, organizational, relational and cognitive- that comprise the potential of a certain territory [traducción de los autores].

${ }^{5}$ The amount and intertwinement of different forms of capital (or different resources) entailed in, mobilized and actively used in (and reproduced by) the regional economy and society [traducción de los autores].

${ }^{6} \mathrm{~A}$ stock of resources specific to the place and available to those who live and work in the territory. These resources (material and immaterial) are common goods for a local community [traducción de los autores].

7 The whole of the local assets which if adequately mobilized provide comparative advantages to those who live and work there [traducción de los autores].
} 
especulativo. Más aún, tales políticas están a menudo fundamentadas en indefendibles supuestos y predicciones no realistas que tratan de valorar las trayectorias futuras del desarrollo económico local"8 (p. 189). De hecho, el premio nobel de economía Paul Krugman ha indicado recurrentemente que la sola noción de competitividad usada para caracterizar de manera aparentemente novedosa las dinámicas actuales del comercio internacional a nivel regional - o como adjetivo de alguna otra cosa- no merece atención alguna. En ese sentido, Brenner y Wachsmuth (2012) consideran que la noción de CT es un pseudoconcepto que "debe ser sistemáticamente decodificada" (p. 197).

A juicio de los autores anteriormente mencionados, esta noción esconde políticas territoriales que generan más efectos negativos que positivos, a saber: a) ahondan los desequilibrios regionales a nivel nacional; b) intensifican la polarización socioespacial; c) agravan los problemas fiscales y regulatorios a nivel municipal; d) minan los activos territoriales en vez de valorizarlos; e) exacerban los problemas de coordinación dentro y entre instituciones del Estado a nivel nacional, regional y municipal, y f) rompen el tejido democrático que permite la toma de decisiones a nivel local por la imposición de lineamientos externos por parte de burócratas, tecnócratas y élites corporativas y empresariales. Así, pues, desde una perspectiva histórica, las políticas de CT van en contravía de las pretensiones que rigieron los lineamientos desarrollistas del territorio y que prevalecieron antes de la década de los setenta: urbanización balanceada, redistribución territorial e igualamiento espacial (Brenner y Wachsmuth, 2012).

\section{Discusión}

Luego de abordar esas dos posiciones antagónicas alrededor del concepto de CT, se plantean unos fundamentos epistemológicos alternativos y preliminares como resultado de la investigación que se viene desarrollado alrededor de la consolidación en la universidad de un OU que aborde este concepto de manera científica y sistemática.

\section{El territorio como univocidad frente al territorio como fragmento}

Un primer elemento para destacar es que en la mayoría de los discursos pro competitividad urbana y regional existe escasa o nula consideración del territorio como unidad. Más bien, estos discursos fragmentan el territorio y lo observan como un simple recipiente que se puede subdividir de una manera económicamente conveniente. Desde ese punto de vista, se entienden las ciudades y regiones como pedazos de territorio propicios para ciertas actividades productivas, pero, en realidad, dichas actividades conforman en sí mismas un sentido único de lo territorial (Tóth, 2014).

Un fundamento epistemológico alternativo para la CT es entender al territorio como univocidad, es decir, encontrar en sus manifestaciones formas de sentido del hacer humano en el espacio. Desde este punto de vista, "un territorio es un espacio social delimitado y lleno de sentidos sociales los cuales implican la operación de poderes sociales relacionales. En cierto sentido el territorio es una expresión de la fusión de significado, poder y espacio social"9 (Delaney, 2009, p. 109). Analíticamente, desde el ámbito de las ciencias humanas es posible considerar que los territorios en cualquiera de sus escalas (nacional, urbana o regional) se constituyen por sí mismos en realidades orgánicas con conciencia independiente. Estas son construcciones sociales y existen en la medida

\footnotetext{
${ }^{8}$ Territorial competitiveness policies have an inherently speculative character [...] Moreover, such policies are often grounded in untenable assumptions and unrealistic predictions regarding the future trajectories of local economic development [traducción de los autores].

${ }^{9}$ A territory is a bounded, meaningful social space the 'meanings' of which implicate the operation of social relational power. It is, in a sense, an expression of the fusion of meaning, power, and social space [traducción de los autores].
} 
en que los humanos las crean. En esa medida, los territorios no pueden ser tratados como empresas, en el sentido de una entidad separada, aunque sí en el sentido weberiano de representar una forma de organización social, es decir, teniendo un propósito y una dirección unificadas, que es lo que define a una empresa en su esencia. Esto último justifica la consideración de que tanto las regiones, ciudades o localidades vistas como organizaciones, puedan competir legítimamente entre ellas en búsqueda de mayor competitividad y atractividad (Capello, Camagni, Chizzolini y Fratesi, 2008).

$\mathrm{Ni}$ siquiera las ciudades que nacieron del sueño de un urbanista o arquitecto ambicioso (Brasilia, Chandigard, etc.) han funcionado siguiendo una dirección definida. Las ciencias sociales han demostrado con suficientes argumentos que las regiones y las ciudades son consecuencia del territorio como un proceso y que, en consonancia con ello, son construcciones históricas inacabadas que cumplen funciones socialmente acordadas por medio de alianzas territoriales locales. En ese sentido, es innegable que "diferentes actores localizados dentro de las ciudades pueden, bajo ciertas condiciones, organizarse colectivamente para promover intereses comunes y agendas"10 (Brenner y Wachsmuth, 2012, p. 194).

\section{De la competencia como correlación simple de fuerzas a la competencia cooperativa}

Es racionalmente admisible considerar que en el proceso de consolidación de los territorios los actores compiten por sus recursos; esta competencia es una realidad social que no solo soporta la esencia de los intercambios altamente especializados que surgieron en los territorios configurados bajo el sistema económico capitalista moderno, sino que está también en la base de la misma supervivencia de los primeros humanos en el planeta tierra. Al respecto, Santos (200) afirma: "en el transcurso de la historia las relaciones entre grupos y, especialmente, los intercambios desiguales, terminaron imponiendo a ciertos grupos las técnicas de otros grupos. Entre aceptación dócil o reticente, entre imposición brutal o disimulada, la elección fue sin embargo inevitable" (p. 160).

Sin embargo, es simple considerar que la lógica de esta lucha que está en la base de la competencia opera en el humano de la misma manera que el resto de los animales y sus estructuraciones territoriales. El uso de la fuerza, así como ocurre con los instintos, está mediado en los humanos por niveles de significación cualitativa y cuantitativamente superiores al de los animales. Eso hace que los procesos de territorialización adquieran diversas formas y que los territorios construidos por actores sociales en los diversos espacios físicos interactúen de forma mayoritariamente cooperativa. Los actos de delimitación e imposición territoriales en humanos no llevan a entidades fragmentadas infalibles e infranqueables, ni siquiera en el caso de las fronteras nacionales. En el caso de los humanos, los dominantes y dominados hacen parte integral de la construcción territorial (Delaney, 2009, p. 198).

A propósito de la competitividad, David Harvey (1989, citado por Brenner y Wachsmuth, 2012), acérrimo crítico del capitalismo, afirma: "una perspectiva crítica sobre el emprenderismo competitivo urbano señala no únicamente sus impactos negativos, sino su potencialidad para su transformación en un corporativismo urbano progresivo"11 (p. 201) Es más, la práctica del planeamiento moderno tanto socialista como capitalista, o incluso aquel que se considere alternativo, mantiene como premisa la competencia en las relaciones humanas. Incluso, la postura más anarquista considera necesario confirmar la competencia como un asunto de correlaciones de fuerza

\footnotetext{
${ }^{10}$ Different actors located within cities may, under certain conditions, organize collectively to promote common interests and agendas [traducción de los autores].

${ }^{11}$ A critical perspective on urban entrepreneurialism indicates not only its negative impacts but its potentiality for transformation into a progressive urban corporatism [traducción de los autores].
} 
en la interacción de los humanos entre ellos y en relación con el espacio que habitan. ${ }^{12}$ De allí se deriva que otro fundamento epistemológico para la CT: tiene que ver la competencia como algo esencialmente cooperativo:

[...] se puede decir que las ciudades y sus regiones se meten a competir con otras localidades únicamente para ampliar las alianzas territoriales ya formadas (ya sea en las escalas local y supra-local), con el propósito explícito de promover una localidad específica como una unidad dentro tal competencia. ${ }^{13}$ (Brenner y Wachsmuth, 2012, p. 196)

No hay competencia entre territorios como tal, sino una ampliación de las alianzas que componen en su conjunto un sistema urbano o regional: "Interlocalidad o competencia territorial es por consiguiente mejor comprendida como una relación horizontal entre crecimiento y alianzas territoriales de inversión orientadas más que una relación vertical entre lugares inmóviles y flujos móviles de capital" (Brenner y Wachsmuth, 2012, p. 184).

\section{De la función económica del territorio a su función social}

El tercer fundamento que se debe reconstruir es la idea dominante de que los territorios persiguen los mismos propósitos y lo hacen de la misma forma. Es cierto que sus agentes internos tienen diferentes intereses y crean diferentes tipos de alianzas. Sin embargo, esto no quiere decir que se imposibilite la existencia de propósitos comunes en el territorio. La inclusión decisiva del territorio como capital dentro de la política económica fue tal vez uno de los grandes logros de las ciencias sociales en la década de los noventa. Algunos académicos de las escuelas de ciencias sociales más importantes del mundo (con preeminencia de la escuela social económica) se reencontraron con la geografía (Krugman, 1995), lo que les permitió actualizar teorías clásicas como la de los factores de localización. Este matrimonio arrojo como resultado que el territorio se introdujera en el complicado y dogmático mundo de la política económica.

La ciencia regional se benefició de ello en el sentido de que contaba con nuevas herramientas analíticas (de orden matemático) que permitían ahora sí modelar las dinámicas de mercado en el espacio (Krugman, 1995). En ese aspecto, el aporte de la masificación de los sistemas computarizados de información geográfica fue clave para sellar una nueva mirada sobre el espacio y la sociedad que interactúa en ella, permitiendo enlazar las variables geográficas a las microeconómicas por medio de sofisticados modelamientos, como aquellos bosquejados por Krugman en Desarrollo, geografía y teoría económica (1995).

Sin embargo y como se ha tratado de demostrar en esta revisión, no se habla en sentido estricto de CT si se siguen atendiendo los factores genéricos de localización de las empresas en el espacio. Se debe dar un paso adicional que nos ubique en la perspectiva de los determinantes territoriales y esté más allá de la mera perspectiva económica de los factores territoriales, así como nos abra posibilidades para el estudio de la CT desde el ordenamiento. Así, los recursos en y del territorio pueden ser de orden material, social y espiritual:

[...] recursos son cosas, naturales o artificiales, relaciones compulsivas o espontáneas, ideas, sentimientos, valores [...] El valor real de cada uno no depende de su existencia separada, sino de su cualificación geográfica, esto es, de la significación conjunta que todos y cada uno obtiene por el hecho de participar de un lugar. (Santos, 2000, pp. 111-112)

\footnotetext{
${ }^{12}$ Algunos consideran que esa perspectiva es ideológica y biologicista, en el sentido que impone como dogma la visión de que "el hombre" actúa como "lobo para el hombre".

${ }^{13} \mathrm{Cities}$ and city regions can be said to engage in interlocality competition only to the extent that territorial alliances are formed -whether at local or supralocal scales- with the explicit goal of promoting a specific locality as a unit within such competition [traducción de los autores].
} 


\section{Conclusiones}

Ante estos escenarios parece que se debiera tomar partido, ya sea por una postura alrededor del concepto de CT más europeísta (continental), basada en la idea de un desarrollo territorial que se soporte en una perspectiva de competitividad; o, más bien, por una postura americana y anglosajona, crítica y aparentemente más rigurosa académicamente, que parece haber dado por clausurada la cuestión con argumentos irrefutables. Sin lugar a dudas, si se toma la segunda opción, se descalificaría desde un punto de vista epistemológico la posibilidad de proponer un futuro OU de la CT en la Fundación Universidad de América.

Estas posturas teóricas motivaron la revisión de la literatura sobre el tema. Ahora bien, una cosa es la CT vista como un mero concepto académico, y otra muy diferente es entenderla como una realidad que, independientemente de sus causas y/o consecuencias, opera como fundamento legitimador de diversas prácticas de ordenamiento territorial. Como académico puro se puede desautorizar la existencia o no de ciertas conceptualizaciones; pero un profesional de una disciplina como la planeación espacial tiene que considerar tanto la dimensión teórica, como la dimensión práctica, por lo que es imperativo sugerir nuevas aproximaciones epistemológicas que, por lo menos, prevean mejores resultados de esas políticas.

Así pues, el proyecto del OU de la Fundación Universidad de América requiere para su formulación de estos nuevos fundamentos, que, además, puedan eventualmente soportar con mayor rigurosidad la incorporación de los principios de CT en las prácticas del ordenamiento territorial. La propuesta se hace desde la disciplina de la planeación espacial, la cual no solo busca criticar y deconstruir los supuestos teóricos sobre los cuales se fundamentan las políticas ordenamiento territorial, sino que también pretende reconstruirlos en función de la necesidad que existe de crear dichas políticas. El OU ofrecerá a los profesionales de la planeación unas herramientas que les permita responder a los problemas generados por las necesidades derivadas del desarrollo territorial; necesidades que, a su vez, surgen de una visión alternativa de la competitividad en los territorios y que no supongan, por un lado, su falsa fragmentación y, por el otro, la mera prevalencia de la competencia económica feroz sobre la cooperación social.

Como lo afirma Toht (2014), el discurso hegemónico de la CT:

[...] no da más detalles y especificaciones de cómo dar forma a las políticas públicas y propuestas de la planeación especial que se inspiran en este concepto problemático [...] tampoco ese discurso ofrece una guía adecuada de cómo aplicar recursos, condiciones y retos mientras se formula un estrategia de desarrollo regional o local en él inspirado"14 (p. 10)

Esta será la misión del OU de la Fundación Universidad de América. También serán objeto de próximas reflexiones las materializaciones que en términos de indicadores e insumos exigen los nuevos principios de CT aquí propuestos.

\section{REFERENCIAS}

Brenner, N., y Wachsmuth, D. (2012). Territorial competitiveness: Lineages, Practices, Ideologies. En B. Sanyal, L. Vale y C. Rosan (Eds.), Planning Ideas That Matter : Livability, Territoriality, Governance, and Reflective Practice (pp. 179-204). Cambridge: MIT Press.

\footnotetext{
${ }_{14}$ Does not give any further details and specifications on how to shape political attitudes and spatial planning approaches [...] did not offer an adequate guidance on how to apply resources, conditions and challenges while developing a local or regional strategy [traducción de los autores].
} 
Buitrago, O. (2016). Competitividad territorial capitalista y despojo del agua en el área de influencia metropolitana de Cali, Colombia. Cuadernos de Geografia: Revista Colombiana de Geografía, 25 (2): 237-250. doi: 10.15446/rcdg.v25n2.54719.

Capello, R., Camagni, R., Chizzolini, B., y Fratesi, U. (2008). Modelling Regional Scenarios for the Enlarged Europe. Berlin, Heidelberg: Springer Berlin Heidelberg. doi:10.1007/978-3-54074737-6

Camagni, R. (2002). On the Concept of Territorial Competitiveness: Sound or Misleading? Urban Studies, 39(13), 2395-2411.

Camagni, R. (2009). Territorial capital and regional development. En R. Capello y P. Nijkamp (Eds.), Handbook of Regional Growth and Development Theories (pp. 118-133). Northampton: Edward Elgar Publishing Limited. Recuperado de http://herzog.economia.unam.mx/ cedrus/descargas/Capello - HB of Regional Growth and Development Theories - 2009.pdf.

Camagni, R., Caragliu, A., y Perucca, G. (2011). Territorial capital: relational and human capital. Recuperado de http://www.grupposervizioambiente.it/aisre/pendrive2011/pendrive/Paper/ Camagni_Caragliu_Perucca.pdf.

Cheshire, P., y Gordon, I. (1998). Territorial competition: Some lessons for policy. The Annals of Regional Science, 32(3), 321-346. doi:10.1007/s001680050077

Delaney, D. (2009). Territory and territoriality. International Encyclopedia of Human Geography, 196-208. doi:10.1016/B978-008044910-4.00808-7

Dimock, M. (1972). Urban Observatories and Managment Strategy. Urban Affairs Review, 8(1), 41-48.

García, R., y Acevedo,J. (2010). El territorio como factor de desarrollo. Semestre Económico, 13(27), 39-62.

Giaime, B. (2011). Weaving the Rural Web: The dynamics of rural development in Lunigiana. Quaderni Sismondi, 12(4), 1-82.

Hawkins, B. (1971) The goal of the urban observatories. The American Review of the Public Administration, 5(2), 113.

Krugman, P. (1995). Desarrollo, geografía y teoria económica. Barcelona: Antoni Bosh.

Murphy, T. (1971) Urban Observatories: Problems and Prospects. The American Review of the Public Administration, 5(2), 131-132.

Organisation for Economic Co-operation and Development. (2001). OECD Territorial Outlook. Paris: OECD. Recuperado de http://www.vwl.tuwien.ac.at/hanappi/AgeSo/SecReps/Territorial_Outlook_F.pdf

Ramírez,J., Osorio, H., y Parra, R. (2007). Escalafón de la competitividad de los departamentos en Colombia. Recuperado de http://repositorio.cepal.org/bitstream/handle/11362/4815/1/S0700110_es.pdf

Ramírez, J., y Alzate, A. (2016). Aproximación a una valoración cienciométrica de la competitividad. Revista de Investigación, 9(1), 121-134.

Ramírez, J., y Alzate, A. (2016). Índices de competitividad: análisis de resultados para Colombia 2015-2016. Revista de Investigación, 9(2), 150-164. 
Sanabria, T., Bolaños, J., y Ramírez, J. (2015). “Ciudades competitivas”. Un panorama conceptual y temático de investigación. Questionar, 3(1), 174-187.

Santos, M. (2000). La naturaleza del espacio. Técnica y tiempo. Razón y emoción (1ª ed.). Barcelona: Editorial Ariel, S. A.

Tóth, B. (2014). Territorial Capital: Theory, Empirics and Critical Remarks. European Planning Studies, 23(7), 1-18. doi.org/10.1080/09654313.2014.928675

Organización de las Naciones Unidas. (2006) A Guide to Setting up an Observatory. Nairobi: UNHabitat.

Van der Ploeg, J., van Broekhuizen, R., Brunori, G., Sonnino, R., Knickel, K., Tisenkops, T., y Oostendie, H. (2008). Towards a framework for understanding regional rural development. En J. van der Ploeg y T. Marsden (Eds.), Unfolding webs: the dynamics of regional rural development (pp. 1-28). Paises Bajos: Royal Van Gorcum. Recueprado de http://orca.cf.ac. $\mathrm{uk} / 20526 /$

Ventura, F., Brunori, G., Milone, P., y Berti, G. (2008). The rural web: a synthesis. En J. van der Ploeg y T. Marsden (Eds.), Unfolding bebs: the dynamics of regional rural development (pp. 149-174). Paises Bajos: Royal Van Gorcum. Recueprado de http://orca.cf.ac.uk/20526/ 
